\title{
Gene expression variation increase in trisomy 21 tissues
}

\author{
Ching Yu Chou $\cdot$ Li Yu Liu • Chien Yu Chen · Cheng Hsien Tsai • \\ Hsiao Lin Hwa $\cdot$ Li Yun Chang · Yi Shing Lin · Fon Jou Hsieh
}

Received: 22 March 2008/Accepted: 27 May 2008/Published online: 2 July 2008

(C) Springer Science+Business Media, LLC 2008

\begin{abstract}
Congenital development disorders with variable severity occur in trisomy 21 . However, how these phenotypic abnormalities develop with variations remains elusive. We hypothesize that the differences in euploid gene expression variation among trisomy 21 tissues are caused by the presence of an extra copy of chromosome 21 and may contribute to the phenotypic variations in Down syndrome. We used DNA microarray to measure the
\end{abstract}

C. Y. Chou

Department of Obstetrics and Gynecology,

Cathay General Hospital, Taipei, Taiwan

L. Y. Liu

Department of Agronomy, Biometry Division,

National Taiwan University, Taipei, Taiwan

L. Y. Liu · C. Y. Chen · F. J. Hsieh

Department of Life Science, National Taiwan University, Taipei,

Taiwan

C. Y. Chen

Department of Bio-industrial Mechatronics Engineering,

National Taiwan University, Taipei, Taiwan

C. H. Tsai

Department of Paediatrics, National Taiwan University Hospital,

Yunlin Branch, Douliou, Taiwan

H. L. Hwa · L. Y. Chang · F. J. Hsieh ( $\square)$

Department of Obstetrics and Gynecology,

National Taiwan University Hospital, Taipei, Taiwan

e-mail: fjhsieh@ntu.edu.tw

\section{Y. S. Lin}

Welgene Biotech. Co., Ltd, 10F-5, No. 3 Yuan-Qu Street,

NanGang Business Park, NanGang, Taipei, Taiwan

\section{F. J. Hsieh}

Center for Systems Biology and Bioinformatics,

National Taiwan University, Taipei, Taiwan differences in gene expression variance between four human trisomy 21 and six euploid amniocytes. The three publicly available data sets of fetal brains, adult brains, and fetal hearts were also analyzed. The numbers of euploid genes with greater variance were significantly higher in all four kinds of trisomy 21 tissues $(p<0.01)$ than in the corresponding euploid tissues. Seventeen euploid genes with significantly different variance between trisomy 21 and euploid amniocytes were found using the $F$ test. In summary, there is a set of euploid genes that shows greater variance of expression in human trisomy 21 tissues than in euploid tissues. This change may contribute to producing the variable phenotypic abnormalities observed in Down syndrome.

\section{Introduction}

Down syndrome (DS) is the most frequent cause of mental retardation. Although a "gene dosage effect" hypothesis has been proposed to explain the effect of the extra chromosome 21 on the pathophysiology of trisomy 21 , it is still a challenge to understand the molecular mechanisms of DS (Antonarakis and Epstein 2006; Roper and Reeves 2006). Elevated levels of gene expression on chromosome 21 in trisomy 21 tissues have been reported (Ait Yahya-Graison et al. 2007; de Haan et al. 2003; Mao et al. 2003; Prandini et al. 2007; von Kaisenberg et al. 1998; Wolvetang et al. 2003). Protein levels of chromosome 21 genes such as APP and SODI are also higher in trisomy 21 tissues (Gulesserian et al. 2001; Mehta et al. 2007). However, Olson et al. (2004) examined chromosome-engineered mice that are trisomy for only the mouse chromosome segment orthologous to the DS critical region (DSCR), but found that the DSCR does not cause 
specific DS phenotypes. It seems that in addition to increased expression of chromosome 21 genes, other factors such as variation in gene expression may also have an important effect on phenotypic presentation in DS.

On the other hand, highly variable phenotypes in DS have long been recognized (Antonarakis and Epstein 2006). Down syndrome patients share some notable phenotypes, but mental retardation, congenital heart disease, and gastrointestinal defects occur with different incidence rates and severity. The underlying mechanism of this phenotypic variability remains obscure. Recently, more and more research has focused on variations in gene expression in trisomy 21 tissues (Ait Yahya-Graison et al. 2007; Deutsch et al. 2005; Prandini et al. 2007; Sultan et al. 2007). It is hypothesized that such variations may result in the phenotypic variability of DS (Prandini et al. 2007). Prandini et al. (2007) categorized human chromosome 21 genes according to the degree of overlap in expression between trisomy 21 and euploid lymphoblastoid cells and fibroblasts. They believe that genes, such as SON and ETS2, with partially overlapping expression distributions between trisomy 21 and normal samples are likely to be involved in the variable features of DS (Prandini et al. 2007). Ait Yahya-Graison et al. (2007) used a specifically designed high-content chromosome 21 microarray to identify genes with differences between trisomy 21 and control lymphoblastoid cells. They found that $15 \%$ of the chromosome 21 genes have highly variable expression among individuals and suggest that these genes, including $A B C G 1$ and $C S T B$, may account for the phenotypic variations observed in patients (Ait Yahya-Graison et al. 2007). Although these two groups have conducted extensive studies of variation in chromosome 21 gene expression, variations in other euploid genes have been ignored. Such variations may also contribute to the phenotypic variability of DS, especially for genes that are involved in the regulation of trisomic genes on chromosome 21.

Recently, microarrays and other high-throughput technologies have allowed steady-state RNA levels to be measured for thousands of transcripts in human trisomy 21 cells (Chung et al. 2005; FitzPatrick et al. 2002; Giannone et al. 2004; Gross et al. 2002; Mao et al. 2003; Tang et al. 2004). Some chromosome 21 genes and non-chromosome 21 genes show overexpression or downregulation in trisomy 21 tissues as compared to normal tissues. Traditionally, these genes are considered to be candidate genes for DS. However, it is possible that some candidate genes such as NFAT (Arron et al. 2006) and downstream genes of NFAT may be expressed with variation and these variations of expression are not obvious in the microarray studies. Instead of analyzing differences of gene expression, investigating the differences of gene expression variation may elucidate phenotypic variation in Down syndrome, since genome-wide analysis of gene expression variation by microarrarys is also widely accepted as a laboratory practice nowadays (Whitehead and Crawford 2006). Therefore, our research aimed at identifying global changes in gene expression and investigating expression variance of euploid genes in trisomy 21 amniocytes using microarrays. In addition, we also analyzed three publicly available microarray data sets of trisomy 21 tissues from GEO (http://www.ncbi.nlm.nih.gov/geo/) and used the same approach to determine whether similar changes in gene expression variation occur in different laboratories using different microarray chips and different tissues.

\section{Materials and methods}

\section{Amniocyte culture}

Primary cultures of amniocytes were collected from fetuses between 16 and 22 weeks of pregnancy, following routine diagnostic cytogenetic analysis of singleton pregnancies. The following cell lines were used in the study: four cases of trisomy 21 ( 2 cases of $47, X X+21$ and 2 cases of 47 , $X Y+21)$ and six cases of euploidy ( 3 cases of $46, X X$ and 3 cases of $46 \mathrm{XY}$ ). The cells were grown in a commercial medium optimized for amniocyte culture at $37^{\circ} \mathrm{C}$ in $5 \%$ $\mathrm{CO}_{2}$. Only culture discs showing normal fibroblast morphology and confluence of more than $80 \%$ of a disc were used for RNA extraction. Informed consent was given by the parents and the study design was approved by the research and ethics committee of the hospital.

\section{Oligonucleotide microarray}

Total RNA was extracted by Trizol ${ }^{\circledR}$ Reagent (Invitrogen, USA), followed by use of an RNeasy Mini Kit (Qiagen, Germany). The purified RNA was quantified by OD260 nm using by a ND-1000 spectrophotometer (Nanodrop Technology, USA), and its quality was assessed by a Bioanalyzer 2100 (Agilent Technology, USA). To serve as reference for microarray comparison, a human reference RNA pooled from ten cell lines (Stratagene, USA) was used. Total RNA $(0.5 \mu \mathrm{g})$ was amplified by a low RNA input fluor linear amp kit (Agilent Technologies, USA) and labeled at $\mathrm{Cy} 3$ or Cy5 (CyDye, PerkinElmer, USA) during in vitro transcription. Tissue RNA was labeled at Cy5, and RNA from Universal Human Reference RNA was labeled at Cy3. Cy-labeled cRNA $(2 \mu \mathrm{g})$ was fragmented to an average size of about 50-100 nucleotides by incubation with fragmentation buffer at $60^{\circ} \mathrm{C}$ for $30 \mathrm{~min}$. Correspondingly fragmented labeled cRNA was then pooled and hybridized to a Human 1A (version 2) oligo microarray (Agilent Technologies, USA) at $60^{\circ} \mathrm{C}$ for $17 \mathrm{~h}$. After washing and drying under a blowing nitrogen gun, the 
microarrays were scanned with an microarray scanner (Agilent Technologies, USA) at $535 \mathrm{~nm}$ for $\mathrm{Cy} 3$ and $625 \mathrm{~nm}$ for Cy5. The scanned images were analyzed by Feature Extraction software (Agilent Technologies, USA). Image analysis and normalization software were used to quantify the signal and background intensities for each feature, and the data were substantially normalized by the rank-consistency-filtering LOWESS method. Finally, the data were presented as $\log 2$. The data discussed in this article have been deposited in the NCBI Gene Expression Omnibus (GEO, http://www.ncbi.nlm.nih.gov/geo/) and are accessible through GEO Series accession number GSE10758. Based on the findings of the MicroArray Quality Control study, a collaborative effort led by the U.S. Food and Drug Administration that included 137 scientists from private and public sector laboratories, they found that microarray gene expression analysis can be used as a standalone quantitative comparison (Shi et al. 2006). Therefore, we did not further reanalyze all genes exhaustively with quantitative polymerase chain reaction.

\section{Statistical methods}

For each array spot, the intensity levels of the two fluorophores were obtained by subtracting the median background intensity from the median foreground intensity. A gene was considered expressed only if the fluorescence intensity of the corresponding spot in foreground signals was at least twice that of the background on every array. For each gene, the logarithm base 2 ratios (referred to henceforth as log ratios) of the two channels were calculated to quantify to the relative expression levels between experimental and reference samples.

The difference of the mean gene expression ratio (trisomy 21 over euploidy) on every chromosome was decided by Student's $t$ test in Excel 2003 ( $p<0.01 / 19840$ with multiple comparisons correction). The distribution of gene numbers with different variance was tested by the $\chi^{2}$ test (STATA 9.0). Other public microarray data sets were searched from GEO (http://www.ncbi.nlm.nih.gov/geo/) using the keywords "homo species" and "Down syndrome." Three public data sets of fetal heart, adult brains, and fetal brains are available online (Conti et al. 2007; Lockstone et al. 2007; Mao et al. 2005). The distribution of gene numbers with different variances was calculated as above and determined to be significantly different by the $\chi^{2}$ test.

The expression of genes that differ among trisomy 21 and euploid amniocytes was evaluated using an ANOVA model. The model can be written as follows:

$Y_{i j}=\mu+\alpha_{i}+\beta_{j}+\varepsilon_{i j}, i=1,2, j=1, \ldots, n$

where $\mu$ is overall mean, $\alpha_{i}$ is the additional effect on gene expression of normal control $(i=1)$ of trisomy $21(i=2)$; $\beta_{j}$ is the expression level of the $j$ th gene, $j=1, \ldots, 21,073$; and $\varepsilon_{i j}$ is the experimental error of the $j$ th gene's expression. It was assumed that the error terms are independently normally distributed with mean 0 and variances $\sigma_{i j}^{2}$. Under the assumption of normality, an $F$ statistic $\left(F_{0 j}\right)$ was adopted to test whether the expressions of the $j$ th gene had equal variance in trisomy 21 and euploid amniocytes (i.e., to test whether $\sigma_{1 j}=\sigma_{2 j}$ ):

$F_{0_{j}}=\frac{S_{T j}^{2}}{S_{C j}^{2}}$

where $S_{C j}^{2}$ is the sample variance of the six expression levels of the $j$ th gene in the euploid group; $S_{T j}^{2}$ is the sample variance of the four expression levels of the $j$ th gene in amniocytes of trisomy 21 . The $F$ statistics follow an $F$ distribution with degrees of freedom 3 and 5 . If $F_{0_{j}}$ yields a very large value, it can be concluded that the expression of the $j$ th gene varies more in trisomy 21 than in euploidy amniocytes. The significance of $F_{0_{j}}$ was determined by estimating the FDR (Storey 2002), which is the proportion of hypotheses among the rejected hypotheses for preselected critical values. To determine the number of significant differences in gene expression variance between the two groups expected by chance if all expression levels were generated randomly, we performed a simulation study with 100 data sets. If all expression levels were generated randomly, the variance of every gene in both groups should be the same and no hypothesis should be rejected by the $F$ test. We performed a simulation with the two groups of samples (4 trisomy versus 6 euploidy control samples) and generated the expression level of every gene by random numbers in the range of -6 to 6 , i.e., the same range in our microarray datasets. The statistical method was the same as the above-mentioned $F$ test and FDR was set at 0.05 .

\section{Clustering analysis of genes on chromosome 21}

The expression activity of genes in euploidy tissues was compared with corresponding reference RNA and relative expression level was defined as the ratio between euploid gene signals and reference RNA signals. That is, if a particular gene is as active as the corresponding reference RNA, the relative expression level (ratio of euploidy genes signal to reference RNA signal) will be around 1 . Otherwise, the relative expression level will be around 0 if the euploid genes' signal is relatively low compared to that of the reference RNA. After filtering the genes for which the corresponding reference RNA gave a low signal (ratio of foreground signal to background signal $<2$ ), the signal intensities of 118 chromosome 21 genes in euploid tissues were analyzed.

The genes were then clustered according to their mean euploid expression and mean expression ratios by 
hierarchical clustering for parameterized Gaussian mixture models (Fraley 1998). The MCLUST (Fraley and Raftery 1999) package was used in R software. An ellipsoidal model with varying volume, shape, and orientation was adopted, and the number of clusters was chosen as four manually.

\section{Results}

Expression of chromosome 21 genes in amniocytes

We measured the expression levels of up to 21,073 transcripts, representing approximately 18,000 genes, using Human 1A (version 2) Oligo Microarray (Agilent Technologies, USA). These transcripts corresponded to 21,073 probe sets, including 100 genes with 10 replicates; therefore, there were 20,173 expression levels of unique genes. After scanning with an Agilent microarray scanner, 322 genes with signal-to-noise ratios less than 2.6 in more than one sample were excluded, leaving 19,840 transcripts for further analysis. In the amniocytes, the mean expression ratio (trisomy 21/euploidy) for genes on individual chromosomes other than 21 was always close to 1 . However, the mean expression ratio for genes on chromosome 21 reached $1.28 \pm 0.06$, significantly different from that of other chromosomes (Fig. $1, p<0.01 / 19,840$ by $t$ test). The confidence interval was also wider for chromosome 21 (Fig. 1). This may be due to a higher variance in expression of chromosome 21 genes.

\section{Analysis of gene expression variances}

After expression variances were calculated for all euploid genes (excluding trisomic genes) in the euploid and trisomy 21 amniocyte microarrays, these genes were stratified by different variance cutoffs, as seen in Fig. 2. We chose a variance of 0.5 as the cutoff for further analysis. Overall, $8.9 \%(1751 / 19,641)$ of euploid genes in trisomy 21 amniocytes had a variance of more than 0.5 compared with

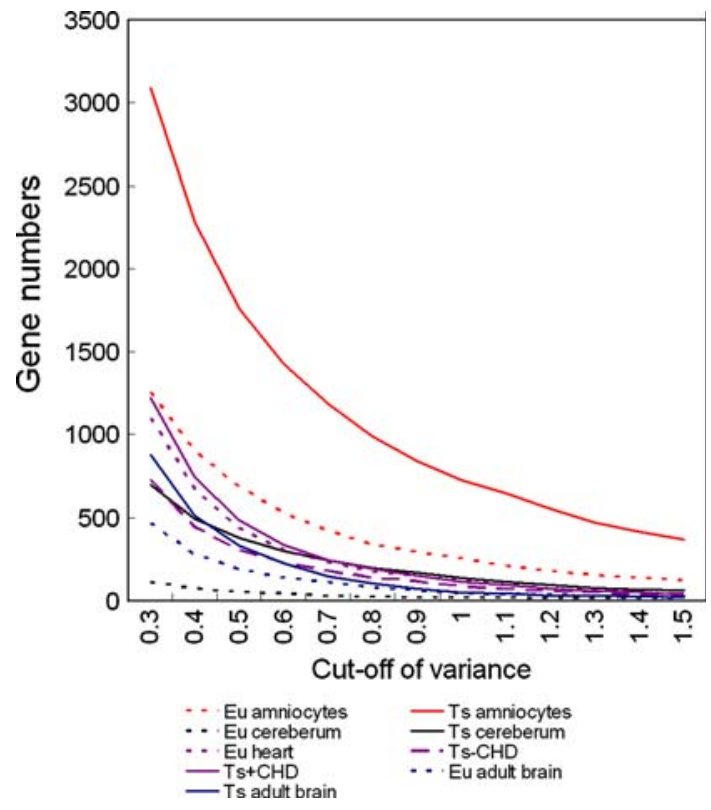

Fig. 2 Gene numbers stratified by different cutoff of variance. The solid lines reveal higher gene numbers of greater variance in trisomy 21 tissues (Ts). The dotted lines reveal lower gene numbers of greater variance in euploid tissues $(\mathrm{Eu})$. In the data sets of fetal hearts, Down syndrome without congenital heart disease (Ts-CHD) reveals the lowest gene numbers of greater variance compared to Down syndrome with congenital heart disease (Ts + CHD). Red line, aminocytes; black line, fetal cereberum; purple line, fetal heart; blue line, adult brain

$2.9 \%(577 / 19,641)$ in euploid amniocytes (Table 1). The number of genes with variance greater than 0.5 was significantly greater in trisomy 21 than euploid amniocytes $\left(p<0.01\right.$, by $\chi^{2}$ test). A leave-one-out analysis was performed in four trisomy 21 amniocytes and six euploid amniocytes. The range of euploid gene numbers (variance $>0.5$ ) was 1013-1751 and 577-665 in three trisomy 21 amniocytes and five euploid amniocytes, respectively. When the same analysis was applied to three other public data sets of microarrays (a fetal brain data set, an adult
Fig. 1 Mean expression ratio (trisomy 21/euploidy) of every chromosome except sex chromosomes. The mean expression ratio of the genes on chromosome 21 reaches $1.28 \pm 0.06$ (bar), while the mean expression ratios of the genes on other chromosomes remain around $1(p<0.01 /$ 19,840). The confidence intervals (whiskers) are also wider in chromosome 21

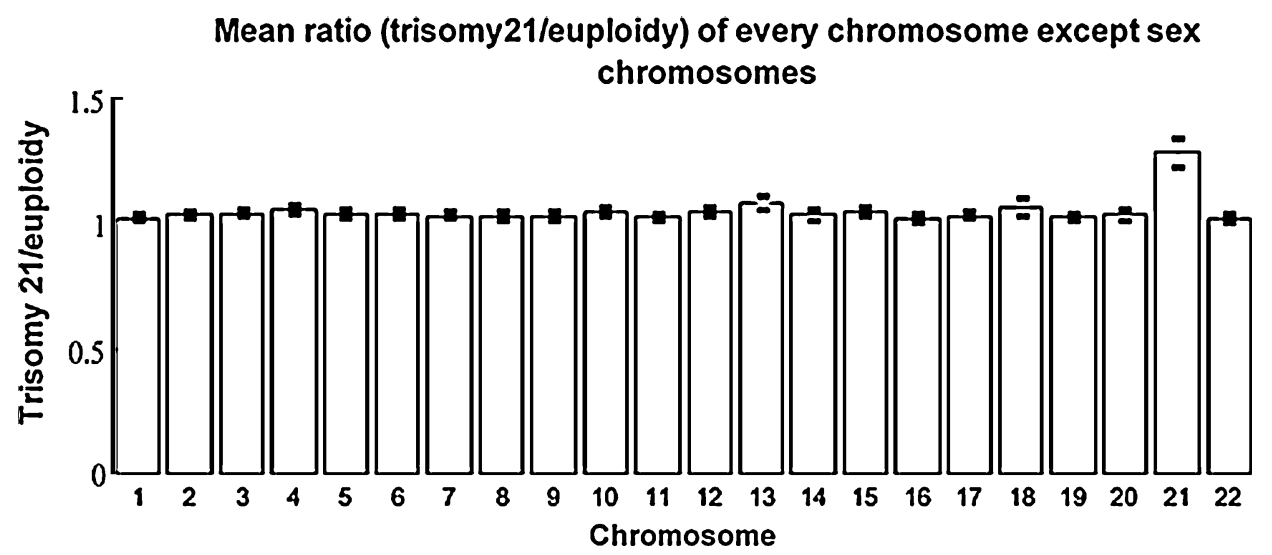


Table 1 Stratification of genes with variance greater than 0.5 in trisomy 21 and euploid tissues

\begin{tabular}{|c|c|c|c|c|}
\hline Tissues & Amniocytes & Fetal hearts & Adults brains & Fetal cerebrums \\
\hline Authors & This study & Conti et al. (2007) & Lockstone et al. (2007) & Mao et al. (2005) \\
\hline GEO series & GSE10758 & GSE1789 & GSE5390 & GSE1397 \\
\hline Genechips & Agilent Human 1A & Affymetrix HG-U133A & Affymetrix HG-U133A & Affymetrix HG-U133A \\
\hline Triosmy 21 & 4 & $\begin{array}{l}5 \text { with } \mathrm{CHD} \\
5 \text { without } \mathrm{CHD}\end{array}$ & 7 & 4 \\
\hline Normal controls & 6 & 5 & 8 & 4 \\
\hline Analyzed probes & 19641 & 7125 & 21989 & 22021 \\
\hline Variances & $<0.5$ & $<0.5$ & $<0.5$ & $<0.5$ \\
\hline Triosmy 21 tissues & $1751 *$ & $\begin{array}{l}6705 \\
6884^{\mathrm{a}}\end{array}$ & 21656 & 21642 \\
\hline Euploidy tissues & 19064 & 6756 & 21800 & 21974 \\
\hline
\end{tabular}

$* p<0.01$

${ }^{\text {a }}$ Trisomy 21 tissues without congenital heart defect

brain data set, and a fetal heart data set), the results were the same (Table 1). In the fetal heart data set, the number of genes with greater variance in trisomy 21 fetuses with congenital heart diseases was much greater than that of trisomy 21 fetuses without congenital heart diseases.

Genes with significantly different expression variances between euploid and trisomy 21 amnioctyes

A total of 42 genes in our amniocyte microarray showed significantly different variances of expression between

Table 2 Euploid gene list with significantly different expression variance between trisomy 21 and euploid amniocytes (pFDR $<0.05$ )

\begin{tabular}{llll}
\hline Gene & pFDR & $\begin{array}{l}\text { Mean expression } \\
\text { of euploidy }\end{array}$ & $\begin{array}{l}\text { Mean expression } \\
\text { of trisomy 21 }\end{array}$ \\
\hline GABRA5 & $2.97 \mathrm{E}-07$ & 0.517632 & 0.854114 \\
GRP & $2.82 \mathrm{E}-06$ & 1.115997 & 2.038489 \\
ZNF84 & $4.6 \mathrm{E}-06$ & 0.737987 & 0.921784 \\
MFAP4 & $6.19 \mathrm{E}-06$ & 0.065456 & 0.100656 \\
MYOG & $8.28 \mathrm{E}-06$ & 1.207202 & 0.779165 \\
ATP5G3 & $1.04 \mathrm{E}-05$ & 1.032876 & 0.819321 \\
KIR2DL5 & $1.56 \mathrm{E}-05$ & 1.139447 & 0.701007 \\
CXCL12 & $1.83 \mathrm{E}-05$ & 0.300409 & 1.12701 \\
SRGAP2 & $2.23 \mathrm{E}-05$ & 1.057018 & 0.632878 \\
KRTHA5 & $3.48 \mathrm{E}-05$ & 1.351911 & 1.843567 \\
C14orf27 & $4.16 \mathrm{E}-05$ & 1.009285 & 1.925189 \\
TAFA5 & $5.77 \mathrm{E}-05$ & 0.139339 & 0.280292 \\
SCAM-1 & $6.14 \mathrm{E}-05$ & 1.448942 & 1.333299 \\
RHCE & $6.59 \mathrm{E}-05$ & 0.099098 & 0.131898 \\
GNLY & $6.61 \mathrm{E}-05$ & 1.239708 & 1.494849 \\
FLJ10637 & $6.7 \mathrm{E}-05$ & 0.484085 & 0.562529 \\
C13orf8 & $8.48 \mathrm{E}-05$ & 0.74312 & 0.959264 \\
\hline
\end{tabular}

pFDR trisomy 21 and euploid amniocytes by the $F$ test when we set FDR to 0.05. Among these genes we excluded those with a foreground-to-background signal ratio of less than 2 and those that are not annotated, leaving a total of 17 genes that met the criteria (Table 2). To determine the number of significant differences in gene expression variance between the two groups that would be expected by chance if all expression levels were generated randomly, we performed a simulation study with 100 data sets. The greatest number proved to be one gene. This indicates that the 42 genes identified in our study are not the result of chance. Searches based on gene ontology (GO) classification indicate that those genes are associated with signal transduction (GO:0007165), cell adhesion (GO:0007155), neuropeptide signaling pathways (GO:0007218), regulation of transcription (GO:0045449), and muscle development (GO:0007517).

\section{Grouping chromosome 21 genes}

We then examined the relationship between the level of expression of chromosome 21 genes and the expression ratio (trisomy 21/euploidy). After filtering the genes for which the corresponding reference RNA had a low signal (ratio of foreground signal to background signal $<2$ ), the signal intensities of 118 chromosome 21 genes in euploidy tissues are analyzed. To elucidate the relationship between expression ratio and expression level for every gene on chromosome 21 , and the different distributions of expression ratio even at similar expression levels, we plotted the expression ratio (trisomy 21/euploidy amniocytes) on the $y$ axis versus the expression level on the $x$ axis. Four clusters of chromosome 21 genes were identified by cluster analysis (Fig. 3): Group I, gene expression in trisomy 21 amniocytes is approximately 1.5-fold higher than in euploid 


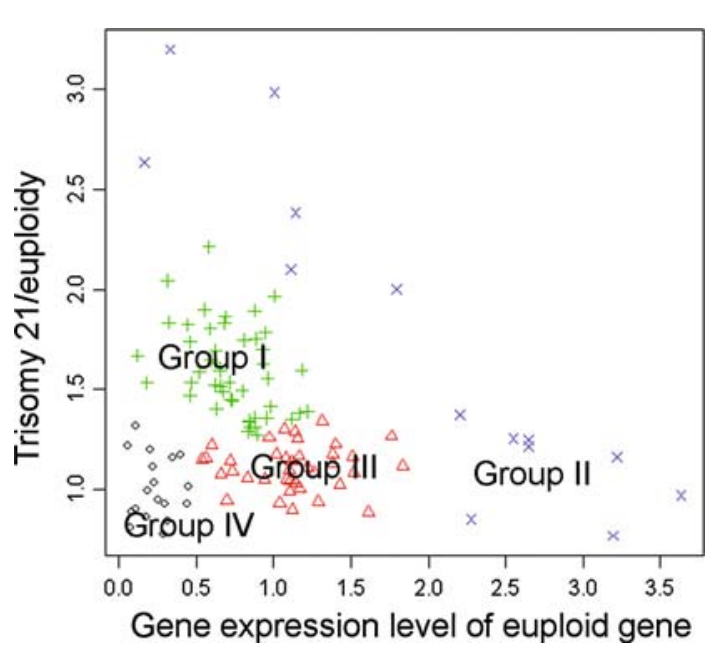

Fig. 3 Relationship between expression ratio (trisomy 21/euploidy) and gene expression level of chromosome 21 genes. To elucidate the differences in distribution of gene expression ratios even when the gene expression levels were similar, the expression ratios (trisomy 21/ euploidy) of each chromosome 21 gene are plotted against its euploid expression level (euploid RNA/reference RNA). Four groups of genes can be identified by clustering analysis as follows: Group I: gene expression in trisomy 21 amniocytes is approximately 1.5 -fold higher than in euploid amniocytes (green crosses). Group II: the gene expression level is amplified or markedly downregulated in trisomy 21 (blue crosses). Group III: gene expression is comparable between euploid and trisomy 21 amniocytes (red triangles). Group IV: gene expression is low in both euploid and trisomy 21 amniocytes (black circles). These four groups of genes are listed. Altogether they constitute the 1.28 -fold change observed for chromosome 21 genes overall in trisomy 21 amniocytes

amniocytes (green crosses); Group II, gene expression is amplified or markedly downregulated in trisomy 21 (blue crosses); Group III, gene expression is comparable between euploid and trisomy 21 amniocytes (red triangles); Group IV, gene expression is low in both euploid and trisomy 21 amniocytes (black circles). Altogether they account for the $1.28 \pm 0.06$ fold change observed for chromosome 21 genes in trisomy 21 amniocytes.

\section{Discussion}

With the advancement of molecular technology, research into Down syndrome is no longer limited to studies of single genes; one can now investigate the expression of all chromosome 21 genes (FitzPatrick et al. 2002; Giannone et al. 2004; Gross et al. 2002; Li et al. 2006; Mao et al. 2003; Prandini et al. 2007; Tang et al. 2004). Two important questions now are: How are these genes expressed differently? and to what extent does variation in expression of these genes differ between trisomy 21 and euploid tissues? Regarding the first question, global upregulation of chromosome 21 genes in Down syndrome has been identified by microarray (Mao et al. 2003;
Prandini et al. 2007; Tang et al. 2004). Prandini et al. (2007) found that the mean overexpression of genes on chromosome 21 in lymphoblastoid cell lines and fibroblasts is 1.44 -fold and 1.67-fold, respectively, with only 39 and $62 \%$ of them overexpressed. Our data also revealed elevated gene expression in trisomy 21 tissues, but there was only a $1.28 \pm 0.06$-fold change on average for chromosome 21 genes (Fig. 1). This may be attributable to differences in gene expression in different tissues and this phenomenon is shown clearly in the Prandini et al. study (2007).

Regarding the second question, previous studies have shown that the variation in expression of some trisomic genes on chromosome 21 increases as the expression level increases (Ait Yahya-Graison et al. 2007; Prandini et al. 2007; Sultan et al. 2007). However, the study of Mao et al. (2005) does not support increasing variation of global gene expression. Saran et al. (2003) performed principal-components analysis on 12 microarray chips (3 Down syndrome mouse with duplicates and 3 euploid mouse with duplicates). They demonstrated greater variability among expression profiles of Down syndrome mouse models (Saran et al. 2003). Also, Potier et al. (2006) found that the number of dysregulated genes changed during postnatal development of the Down syndrome mouse. In this study, we found that the number of euploid genes with variance greater than 0.5 was significantly higher in trisomy 21 tissues than in the euploid equivalents. Also we used the $F$ test to find the genes with significantly increased variation in trisomy 21 tissues. However, it is still hard to estimate the number of genes with different variance and how much of variance is enough for a biological significance since we still know little about gene expression variation and associated presentation.

Ait Yahya-Graison et al. (2007) classified genes on chromosome 21 into four groups according to their expression ratios and variation in gene expression. Although we used a different approach, we found a similar biological meaning for these classes. In their study, Class I genes had DS/control expression ratios close to 1.5 , consistent with our Group I genes. Their Class II contained genes with expression ratios greater than 1.64 and forms part of our Group II. Class III genes had ratios less than 1.4 and compensation was suspected. These genes are close to our Group III genes. Their Class IV genes showed highly variable presentation and would be dispersed among our Groups I-III. In addition, we found a subset of Group IV genes that show low activity in trisomy 21 and euploid amniocytes. Although their trisomy 21/euploidy ratios are close to 1.0, their gene expression levels are low in both types of amniocytes. They may have different expression ratios in other tissues but not in amniocytes. Therefore, our gene expression ratios for these genes are inconclusive. 
Gene expression variability was affected by stochastic genetic effects and genetic and genomic differences (Cheung et al. 2003). Since the propagation of variation in gene networks has been demonstrated by single-cell studies and model simulations (Pedraza and van Oudenaarden 2005), it is reasonable to suppose that a downstream gene in a pathway circuit may inherit and accumulate its amplitude of variation from upstream trisomic genes, and the downstream genes on chromosomes other than 21 may show the greatest variance. If we consider stochastic genetic effects as a contributing factor and assume that genetic difference is impossible to be controlled in humans but is possible in animal models, we propose two possible mechanisms for the increased expression variance of these euploid genes. We assumed that Group I and Group II chromosome 21 genes respond to an extra copy of chromosome 21 , inducing a 1.5 -fold or greater change. Thus, it is possible that variance of expression of these genes increases as the expression level increases. The variance of downstream euploid genes may be amplified by pathway propagation from Group I and Group II genes. Perhaps these euploid genes inherit the greatest variance, which is more evident from the $F$ test, than genes on chromosome 21 . That is why all the genes extracted by the $F$ test come from chromosomes other than 21 . We also assumed that Group III chromosome 21 genes are expressed at the same levels in both euploid and trisomy 21 amniocytes. Tight negative feedback mechanisms may maintain this constancy, even in the presence of an extra chromosome 21. Contrary to the view of Ait Yahya-Graison et al. (2007), we consider that Group III genes also partially contribute to the variation in expression of euploid genes. In trisomy 21 tissues, the final concentration of Group III genes may reach the threshold early and expression is inhibited until the concentration of product is low enough to reactivate it. Thus, compared to euploid tissues, the transcripts measured at the time of sampling show more variance in trisomy 21 tissues because their expression is in an unstable condition or is oscillating between activation and inhibition. This instability may propagate to downstream euploid genes. However, It more evidence is needed before deciding if variability of phenotype came from originally stochastic genetic expression. Even more, the associated anomaly resulted from exaggerated variation caused by an extra chromosome 21.

In summary, the mean expression level of genes on chromosome 21 in trisomy 21 amniocytes is significantly higher than that of genes on all other chromosomes. A set of euploid genes shows greater variance in gene expression in human trisomy 21 tissues than in euploid tissues. Instead of analyzing differences of gene expression, investigation of the differences of gene expression variation may better elucidate phenotypic variation of Down syndrome.
However, more evidence is needed to decide whether the associated anomaly results from increased variation caused by an extra chromosome 21 .

Acknowledgments The authors thank Dr. Chen Chih-Ping and Dr. Tsang-Ming Ko for providing trisomy 21 amniocytes. This work was supported by National Science Council (NSC) grant No. 95-2314-B002-255-MY3.

\section{References}

Ait Yahya-Graison E, Aubert J, Dauphinot L, Rivals I, Prieur M et al (2007) Classification of human chromosome 21 gene-expression variations in Down syndrome: impact on disease phenotypes. Am J Hum Genet 81:475-491

Antonarakis SE, Epstein CJ (2006) The challenge of Down syndrome. Trends Mol Med 12:473-479

Arron JR, Winslow MM, Polleri A, Chang CP, Wu H et al (2006) NFAT dysregulation by increased dosage of DSCR1 and DYRK1A on chromosome 21. Nature 441:595-600

Cheung VG, Conlin LK, Weber TM, Arcaro M, Jen KY et al (2003) Natural variation in human gene expression assessed in lymphoblastoid cells. Nat Genet 33:422-425

Chung IH, Lee SH, Lee KW, Park SH, Cha KY et al (2005) Gene expression analysis of cultured amniotic fluid cell with Down syndrome by DNA microarray. J Korean Med Sci 20:82-87

Conti A, Fabbrini F, D’Agostino P, Negri R, Greco D et al (2007) Altered expression of mitochondrial and extracellular matrix genes in the heart of human fetuses with chromosome 21 trisomy. BMC Genom 8:268

de Haan JB, Susil B, Pritchard M, Kola I (2003) An altered antioxidant balance occurs in Down syndrome fetal organs: implications for the "gene dosage effect" hypothesis. J Neural Transm Suppl 67:67-83

Deutsch S, Lyle R, Dermitzakis ET, Attar H, Subrahmanyan L et al (2005) Gene expression variation and expression quantitative trait mapping of human chromosome 21 genes. Hum Mol Genet 14:3741-3749

FitzPatrick DR, Ramsay J, McGill NI, Shade M, Carothers AD et al (2002) Transcriptome analysis of human autosomal trisomy. Hum Mol Genet 11:3249-3256

Fraley C (1998) Algorithms for model-based Gaussian hierarchical clustering. SIAM J Sci Comput 20:270-281

Fraley C, Raftery AE (1999) MCLUST: software for model-based cluster analysis. J Classification 16:297-306

Giannone S, Strippoli P, Vitale L, Casadei R, Canaider S et al (2004) Gene expression profile analysis in human T lymphocytes from patients with Down Syndrome. Ann Hum Genet 68:546-554

Gross SJ, Ferreira JC, Morrow B, Dar P, Funke B et al (2002) Gene expression profile of trisomy 21 placentas: a potential approach for designing noninvasive techniques of prenatal diagnosis. Am J Obstet Gynecol 187:457-462

Gulesserian T, Seidl R, Hardmeier R, Cairns N, Lubec G (2001) Superoxide dismutase SOD1, encoded on chromosome 21, but not SOD2 is overexpressed in brains of patients with Down syndrome. J Investig Med 49:41-46

Li CM, Guo M, Salas M, Schupf N, Silverman W et al (2006) Cell type-specific over-expression of chromosome 21 genes in fibroblasts and fetal hearts with trisomy 21. BMC Med Genet $7: 24$

Lockstone HE, Harris LW, Swatton JE, Wayland MT, Holland AJ et al (2007) Gene expression profiling in the adult Down syndrome brain. Genomics 90:647-660 
Mao R, Zielke CL, Zielke HR, Pevsner J (2003) Global up-regulation of chromosome 21 gene expression in the developing Down syndrome brain. Genomics 81:457-467

Mao R, Wang X, Spitznagel EL Jr, Frelin LP, Ting JC et al (2005) Primary and secondary transcriptional effects in the developing human Down syndrome brain and heart. Genome Biol 6:R107

Mehta PD, Capone G, Jewell A, Freedland RL (2007) Increased amyloid beta protein levels in children and adolescents with Down syndrome. J Neurol Sci 254:22-27

Olson LE, Richtsmeier JT, Leszl J, Reeves RH (2004) A chromosome 21 critical region does not cause specific Down syndrome phenotypes. Science 306:687-690

Pedraza JM, van Oudenaarden A (2005) Noise propagation in gene networks. Science 307:1965-1969

Potier MC, Rivals I, Mercier G, Ettwiller L, Moldrich RX et al (2006) Transcriptional disruptions in Down syndrome: a case study in the Ts1Cje mouse cerebellum during post-natal development. J Neurochem 97(Suppl 1):104-109

Prandini P, Deutsch S, Lyle R, Gagnebin M, Delucinge Vivier C et al (2007) Natural gene-expression variation in Down syndrome modulates the outcome of gene-dosage imbalance. Am J Hum Genet 81:252-263

Roper RJ, Reeves RH (2006) Understanding the basis for Down syndrome phenotypes. PLoS Genet 2:e50
Saran NG, Pletcher MT, Natale JE, Cheng Y, Reeves RH (2003) Global disruption of the cerebellar transcriptome in a Down syndrome mouse model. Hum Mol Genet 12:2013-2019

Shi L, Reid LH, Jones WD, Shippy R, Warrington JA et al (2006) The MicroArray Quality Control (MAQC) project shows inter- and intraplatform reproducibility of gene expression measurements. Nat Biotechnol 24:1151-1161

Storey JD (2002) A direct approach to false discovery rate. J Royal Statistical Soc B 64:479-498

Sultan M, Piccini I, Balzereit D, Herwig R, Saran NG et al (2007) Gene expression variation in 'Down syndrome' mice allows to prioritize candidate genes. Genome Biol 8:R91

Tang Y, Schapiro MB, Franz DN, Patterson BJ, Hickey FJ et al (2004) Blood expression profiles for tuberous sclerosis complex 2 , neurofibromatosis type 1, and Down's syndrome. Ann Neurol $56: 808-814$

von Kaisenberg CS, Brand-Saberi B, Christ B, Vallian S, Farzaneh F et al (1998) Collagen type VI gene expression in the skin of trisomy 21 fetuses. Obstet Gynecol 91:319-323

Whitehead A, Crawford DL (2006) Variation within and among species in gene expression: raw material for evolution. Mol Ecol 15:1197-1211

Wolvetang EJ, Bradfield OM, Hatzistavrou T, Crack PJ, Busciglio J et al (2003) Overexpression of the chromosome 21 transcription factor Ets2 induces neuronal apoptosis. Neurobiol Dis 14:349-356 